\title{
Cortesía y urbanidad en los libros de civismo lasallistas mexicanos (1953-1989)
}

\section{Courtesy and civility in the mexican Lasallian civic books (1953-1989)}

\author{
Larisa González Martínez \\ Universidad Iberoamericana León (México)
}

Recibido: 25 de julio de 2020 Aceptado: 12 de febrero de 2021 Publicado: 31 de mayo de 2021

\section{Resumen}

El artículo busca explicar la formación cívica que los Hermanos de la Salle impartieron en México entre 1953-1989, mediante el análisis de los manuales escolares de Bernardo Zepeda Sahagún. Como resultado de la investigación, se pone en evidencia que la cortesía y las buenas maneras son elementos primordiales del civismo de los religiosos lasallistas debido a su espiritualidad y la influencia de ideas provenientes de lo que sería el Catecismo y de algunos fragmentos bíblicos. El estudio además, concluye que los libros de texto lasallistas se propusieron desarrollar la virtud (formar buenos cristianos) y la ciudadanía, por lo que se propone la categoría de la persona cívica.

Palabras clave: Hermanos de las Escuelas Cristianas; libros de texto; Congregaciones educadoras; Historia de la Educación; Civismo

”Email: larisa.gonzalez.martinez@hotmail.com 


\section{Abstract}

The article seeks to explain the civic training that the De la Salle Brothers imparted in Mexico between 1953-1989, through the analysis of Bernardo Zepeda Sahagún's textbooks. As a result of the investigation, it is evidenced that courtesy and good manners are essential elements of civility of the lasallian religious due to their spirituality and the influence of ideas coming from what would be the Catechism and some biblical fragments. The study also concludes that Lasallian textbooks set out to develop virtue (to form good Christians) and citizenship, which is why the category of the civic person is proposed.

Keywords: Brothers of the Christian Schools; textbooks; Teaching congregations; History of education; Civics. 


\section{Introducción}

El presente artículo tiene como objetivo explicar la formación cívica que los Hermanos de la Salle impartieron en México entre 1953-1989, mediante el análisis de los manuales escolares del Hermano Bernardo Zepeda Sahagún (con influencias como los elementos dogmáticos de lo que sería el Catecismo, la Biblia y la cortesía y la urbanidad de la espiritualidad lasallista) cuyo propósito era formar buenos cristianos que, en el futuro, debían ser correctos ciudadanos.

Para ello, en un primer momento se ofrecerá al lector un marco categorial conformado por los términos civilización de Norbert Elias y buenas maneras de Anna Bryson. Se ha elegido a estos autores porque sus nociones permiten establecer una serie de elementos que propician la revisión de la obra de Jean-Baptiste de la Salle y el análisis de los libros de texto de civismo publicados por los Hermanos en México, como la existencia de una intención por regular la conducta cuya expresión concreta son las buenas maneras, las cuales se promueven desde el ámbito educativo a través de sus diversas formas.

Posteriormente, se hará una revisión de las ideas sobre la cortesía y la urbanidad presentes en la obra de Jean-Baptiste de la Salle, que abrirán paso a un apartado metodológico centrado en el Modelo Tridimensional de Análisis Crítico del Discurso de Norman Fairclough. Finalmente, el lector encontrará un breve contexto sobre la editorial responsable de los libros de los Hermanos de las Escuelas Cristianas en México, seguido del análisis de los manuales escolares de civismo que los lasallistas publicaron en este país entre los años 1953-1989.

Para este último aspecto del presente trabajo de investigación se lleva a cabo un proceso de descripción de los libros elegidos, seguido de un estudio de los "procesos de producción e interpretación de los textos". Esto último quiere decir, que se hará énfasis en las características de los libros de clase lasallistas como herramienta pedagógica y cognoscitiva, además de que se pondrá atención en los atributos de los destinatarios de estos manuales escolares, es decir, los alumnos.

Para completar el estudio de los manuales escolares de civismo de acuerdo con el Modelo Tridimensional de Análisis Crítico del Discurso de Norman Fairclough se buscará comprender su carácter situacional e institucional rastreando la influencia de la Iglesia (y la religión) Católica y la espiritualidad de Jean-Baptiste de la Salle. Para ello se señalan los textos que sirven de fuentes para las afirmaciones contenidas en los manuales escolares, se evidencian algunas ideas de los lasallistas en México sobre la formación del alumno en la cortesía y la urbanidad y se enumeran los atributos del estudiante que se buscaba formar.

\section{Civilización y buenas maneras como categorías para el análisis}

Norbert Elias propuso que la civilización es un proceso que "supone una transformación del comportamiento y de la sensibilidad humanos en una dirección determinada", lo cual significa "un control emotivo más fuerte y más proporcionado" (2016, pp. 35, 535). Cabe señalar que la vergüenza y la racionalización del comportamiento, la coacción social e institucional (a través de organizaciones fuertes que monopolizan, centralizan y administran la violencia física) y la autocoacción fueron elementos en juego para Elias en este cambio de comportamientos y sensibilidades sociales y personales (2016, pp. 535-542, 593). 
Esta intención por regular la conducta permea el discurso educativo, como el de la obra de Jean-Baptiste de la Salle destinada a la cortesía y la urbanidad, o el de los libros de civismo de los lasallistas en México. Además, a través de este tipo de discurso se propagan las "representaciones sociales básicas de los grupos sociales" (las ideologías) que "contienen los principios básicos que organizan las actitudes que comparten los miembros de un grupo" (Van Dijk, 2003, p. 170). Hay que subrayar que esta "manera particular de representar y construir la sociedad" (Fairclough y Wodak, 2000, p. 392) propicia, a su vez, la reproducción de esquemas, la continuidad de normas y otros fenómenos no menos interesantes.

El análisis de la sociedad cortesana francesa durante el absolutismo monárquico, otorgó a Norbert Elias la posibilidad de visualizar el control de los afectos como un elemento de competencia. Es decir que el autocontrol y la disciplina personal junto con la sumisión de la apariencia y la conducta a las normas establecidas, se transforman en vías para el progreso social, o la conservación del prestigio (Elias, 2012, pp. 146, 284, 321).

Norbert Elias consideraba que la civilización es un valor particular, relativo históricamente, que apareció en un período y un lugar concretos: Europa, entre la Edad Media y la Modernidad (Torres Septién, 2002, p. 356). Esto y sus investigaciones sobre la sociedad cortesana en Francia invitan a considerar seriamente el peso del absolutismo francés en el contexto de Jean-Baptiste de la Salle, lo cual puede ayudar a explicar su pensamiento en el que la cortesía y la urbanidad tuvieron un lugar importante. Este último aspecto, a su vez, permearía la obra de los religiosos lasallistas con el paso de los años.

En este punto, resulta necesaria la definición de las buenas maneras, que son un "conjunto de reglas sociales" que marcan la pauta sobre nociones que resultan cruciales para vivir en sociedad, como lo "vergonzoso", lo "grotesco", lo "asqueroso", lo "incorrecto", lo "refinado", lo "decente" y lo "indecente" (Bryson, 2002, p. 367). Las buenas maneras delimitan los ideales o modelos de conducta que se consideran deseados o correctos para un grupo de individuos (Torres Septién, 2002, p. 360), los cuales se manifiestan en aspectos como "las formas de vestir, el trato con los demás y el comportamiento" (Bryson, 2002, p. 368). Hay que subrayar que las buenas maneras se considerarán aquí como la expresión práctica e inmediata de un comportamiento civilizatorio, además de que cumplen también la función de eliminar o reducir las tensiones que se producen en la convivencia social (Calsamiglia Blancafort y Tusón Valls, 1999, p. 162).

\section{La cortesía y la urbanidad en la obra de Jean-Baptiste de la Salle}

Reglas de cortesía y urbanidad cristiana para uso de las escuelas cristianas es un libro escrito por Jean-Baptiste de la Salle, para su uso por alumnos en el octavo nivel de lectura. Con el tiempo, este texto llegó a otros públicos (y a otros lugares fuera de Francia), trascendiendo el ámbito para el que había sido creado (De la Salle, 2001c, pp. 193-195).

Ciertamente, la obra de la Salle dedicada a la cortesía y la urbanidad no fue un fenómeno aislado en su contexto. Para explicarlo, es necesario dirigir la mirada al siglo XVI, cuando la Reforma protestante se desarrolló en Europa Occidental (López Sánchez, 2009, p. 183). El impacto de este fenómeno religioso, social e, incluso, político, se dejó sentir en diversos aspectos de la vida, entre ellos, el de los comportamientos, a través del surgimiento de una moral y una ética protestantes. 
Esto último ocurrió como resultado de un cambio profundo en las creencias relativas a la salvación. Así, mientras que para la fe católica las buenas obras son necesarias para alcanzarla, los protestantes asumían que se llegaba a ésta mediante la gracia divina. Esto ocasionó que el creyente asumiera una actitud de autovigilancia permanente, al grado de desarrollarse un ascetismo secular, complementado con el control social mutuo. La vigilancia por parte de los demás surgió, a su vez, por otros cambios introducidos por el protestantismo. Uno de ellos fue la supresión del sacramento de la penitencia, muy ligado a la idea de que la Iglesia es intermediaria entre Dios y los seres humanos. En este sentido no hay que olvidar que, de acuerdo con las iglesias protestantes, la Biblia es la única fuente de revelación y se encuentra al alcance de todos los fieles (Arruñada, 2010, p. 894).

La supervisión de la propia conducta y de la de los demás que fue resultado del protestantismo, se manifestó con el surgimiento de una moral y una ética cuya influencia se hizo sentir en el ámbito de lo personal y privado pero, también, en el universo de lo político y lo económico (Cervantes-Ortiz, 2007, pp. 40-45). Así, los comportamientos, los hábitos laborales, los gestos del cuerpo y otros aspectos, comenzaron a ser fiscalizados, una conducta que no pasó desapercibida para la Iglesia católica.

Al inicio de las Reglas de cortesía y urbanidad cristiana para uso de las escuelas cristianas, Jean-Baptiste de la Salle manifiesta su inconformidad con aquellos que asumen que la urbanidad y la cortesía son cualidades meramente humanas y mundanas pues, para este autor, ambas obedecen a una motivación cristiana en esencia. Por tanto, para la Salle es crucial que los adultos no inviten a los niños a seguir las reglas de las buenas maneras alegando que, de no cumplirlas, "se los criticará, perderán la estima o se los ridiculizará, ya que todas esas formas sólo son adecuadas para inspirarles el espíritu del mundo y para alejarlos del espíritu del Evangelio" (De la Salle, 2001c, p. 197).

Cabe mencionar que, de acuerdo con el fundador de los Hermanos de la Salle, la cortesía y la urbanidad son también aspectos de la virtud de la caridad, especialmente cuando se trata del amor hacia los demás, manifiesto en las prácticas de urbanidad vinculadas con el prójimo a través de expresiones de benevolencia y honor. Aún más, de acuerdo con el autor, estas manifestaciones de respeto no deben originarse por la deferencia hacia los nombramientos y rangos, sino por la naturaleza de las personas como miembros de Cristo y templos vivos movidos y alentados por el Espíritu Santo (De la Salle, 2001c, pp. 197-198).

¿Debe considerarse que, a través de estas palabras, Jean-Baptiste de la Salle desestimaba el peso de la coacción social e institucional y del prestigio social en la regulación de la conducta? Considerando el contexto en el que el fundador de los lasallistas vivió (el monarquismo absolutista de la Francia del Rey Sol, quien instituyó una complicada etiqueta que daba sentido a la vida social) no es posible afirmar esto, aunque para este autor también existe otra motivación para la ordenación del comportamiento.

Ciertamente, como se vio con ayuda de las categorías de este texto, cuando se trata del comportamiento civilizatorio y las buenas maneras, la presencia física, la apariencia y la conducta están sujetas a la vigilancia y la aprobación de otros. Esto significa que el individuo está constantemente siendo observado y juzgado, por lo que se ve obligado a seguir fielmente las disposiciones dictadas, para así evitar la burla, la desaprobación y el escarnio. 
En el caso de la propuesta de conducta elaborada por Jean-Baptiste de la Salle, es posible establecer que para él otro observador que evalúa los comportamientos es Dios, por lo que su mirada divina se posa sobre la persona permanentemente para evaluar sus acciones. Es por esta razón que la idea de la Santa Presencia de Dios está tan presente en la espiritualidad y la pedagogía lasallistas. De hecho, este pensamiento está presente en la obra del fundador de los religiosos de la Salle, concretamente, en la Guía de las Escuelas: "Se les animará a que entren en sus clases con profundo respeto, en atención a la presencia de Dios".

\section{O bien:}

Los que leen en el segundo cartel aprenderán y repetirán los actos de la presencia de Dios, de invocación al Espíritu Santo, de adoración y de agradecimiento, que vienen a continuación del comienzo tanto de la oración de la mañana como de la tarde.

Y también:

Repasarán la oración de la siguiente manera: uno dice una frase y otro la siguiente. El primero, por ejemplo, dirá: Acordémonos de que estamos en la presencia de Dios, y digamos; después añadirá: Dios mío, creo firmemente que estáis en todas partes y que estáis aquí presente. El otro seguirá: que me veis y que me oís. El primero dirá luego: Creo que nada hay oculto para vos, y que conocéis todos mis pensamientos y lo profundo de mi corazón.

Finalmente:

A cada hora del día se harán breves oraciones que servirán al maestro para renovar su atención sobre sí mismo y a la presencia de Dios, y a los escolares para habituarles a pensar en Dios de vez en cuando durante el día, y disponerlos a ofrecerle todas sus acciones, para atraer su bendición sobre ellas (De la Salle, 2001, pp. 13, 17-18, 51).

Hay que decir que la propuesta de Jean-Baptiste de la Salle establece la existencia de dos tipos de cortesía y urbanidad: una "puramente mundana y casi pagana" y otra cristiana (De la Salle, 2001c, p. 198). Evidentemente, de la Salle considera que sus ideas pertenecen a la segunda categoría. Por ello, la cortesía y la urbanidad cristianas son definidas por este autor como "un proceder prudente y regulado que uno manifiesta en sus palabras y acciones exteriores, por sentimiento de modestia, de respeto, o de unión y caridad para con el prójimo, y toma en consideración el tiempo, los lugares y las personas con quienes se trata" (De la Salle, 2001c, p. 198).

Para afinar esta breve exposición de las Reglas de cortesía y urbanidad cristiana, cabe señalar que la disposición de los temas en el libro se desarrolla en dos grandes ejes temáticos. El primero de ellos se enfoca en el recato que debe observarse en los modales y en el comedimiento del cuerpo, mientras que el segundo habla de 
las señales de respeto hacia los demás ante diversas situaciones de carácter social (De la Salle, 2001, p. 199).

En el primero de estos apartados se habla sobre la postura, el autodominio y la compostura del cuerpo y sus movimientos, un tema tan importante para Jean-Baptiste de la Salle que es tratado en otras de sus obras. Un ejemplo de ello son las Reglas comunes de los Hermanos de las Escuelas Cristianas que establecen que los religiosos lasallistas "llevarán siempre la cabeza derecha, inclinándola un poco hacia adelante; no la volverán atrás, ni de un lado a otro; y si la necesidad les obliga a ello, volverán a un tiempo todo el cuerpo pausada y gravemente" (De la Salle, 2001b, p. 35). Asimismo, La Guía de las Escuelas Cristianas establece que el profesor deberá vigilar que:

Los alumnos tengan el cuerpo lo más derecho posible, y que no lo inclinen más que un poco, sin tocar la mesa, de modo que teniendo apoyado el codo en la mesa, puedan apoyar la barbilla en el puño. Deben tener el cuerpo ligeramente vuelto y libre hacia el lado izquierdo, de manera que todo el peso recaiga sobre este lado. El maestro exigirá que mantengan exactamente todas las posturas que se refieren a la posición del cuerpo, tal como están indicadas en las normas de la escritura.

Cuidará, sobre todo, de que no separen demasiado del cuerpo el brazo derecho, y que no apoyen el estómago en la mesa. Pues, aparte de que esta postura es muy desagradable, les podría causar graves molestias.

Para hacer que mantenga bien el cuerpo, el maestro pondrá él mismo al alumno en la postura que debe tener. Para este fin, le colocará cada miembro en el lugar en que debe estar y, cuando vea que cambia de postura, cuidará de volver a colocarlo bien (De la Salle, 2001, pp. 40-41).

Los aspectos relacionados con el autodominio, la compostura y los movimientos permiten vislumbrar también la presencia del concepto de la persona humana en las concepciones sobre el cuerpo que el fundador de los Hermanos expone en su obra. Y es que, al resaltar que el hombre es hijo de Dios y, por tanto, ha sido hecho a su imagen y semejanza, su cuerpo participa de la dignidad de la imagen del Creador, de lo cual se deriva el necesario respeto y vigilancia que debe tributársele. Posteriormente, cuando en el texto se habla sobre las partes del cuerpo "que se deben mantener ocultas", se vuelve sobre la idea de que los cuerpos son "templos vivos, en los que Dios quiere ser adorado en espíritu y en verdad" o "tabernáculos que Jesucristo se ha escogido como morada" (De la Salle, 2001c, p. 216).

Otros temas también presentes en la primera parte de las Reglas de cortesía y urbanidad cristiana para uso de las escuelas cristianas son diversas recomendaciones sobre las diferentes partes del cuerpo como la cabeza, las orejas, el rostro, los ojos, la nariz, la lengua, los labios, los dientes y la boca.

El Fundador de los Hermanos aconseja desarrollar un tono de voz suave y apacible (De la Salle, 2001c, p. 210), con un volumen adecuado que permita ser escuchado sin gritar pues, alzar la voz es especialmente molesto. Es por esta razón que para el autor es una mala costumbre que debe evitarse 
en los estudiantes, tal y como se lee en la Guía de las escuelas, en la cual se advierte, por ejemplo, que los alumnos no deben esperar a la entrada de la escuela gritando o cantando (De la Salle, 2001, p. 12).

Finalmente, debe decirse que en el segundo apartado de la obra lasaliana dedicada a la cortesía y la urbanidad se hacen recomendaciones sobre los momentos y las formas en los que una persona debe acostarse y levantarse, la elección de la vestimenta, el momento de tomar alimentos, las diversiones, la conversación, la manera en la que hay que dar y recibir objetos, los modos de comportarse con las personas cuando se les encuentra de manera casual, las formas en las que hay que conducirse en la calle o en los viajes a caballo y en carroza, además de algunas instrucciones sencillas para escribir cartas con corrección.

\section{Un contexto histórico necesario: Las editoriales lasallistas en México}

Para contextualizar el fenómeno estudiado es necesario aludir a las dos editoriales lasallistas que se fundaron en México: Enseñanza y Didáctica. La primera de ellas es la más antigua de las iniciativas editoriales propiamente de los Hermanos de las Escuelas Cristianas en México, aunque datar su fundación es una cuestión complicada.

Para hacerlo, hay que comenzar por señalar que los primeros libros de clase lasallistas fueron traducciones de los manuales escolares que los Hermanos publicaron en Europa a través de la Procure Générale (Procura de París) y que fueron editados en México por la Casa Bouret, representada por Raoul Mille. Esta primera etapa editorial lasallista (entre 1909 y 1912) -y en la que se elaboraron libros teniendo como autor a G.M. Bruño y a la Casa Bouret como editor- (Grousset y Meissonier, 1983a, pp. 86-88) pronto dio paso a la creación de una editorial propia de los religiosos de la Salle (Enseñanza), que pudo surgir entre 1930 y 1940.

Ante la imposibilidad de consultar el acta constitutiva de esta editorial, que legalmente debería considerarse como su nacimiento por la vía jurídica (por lo menos de acuerdo con la Ley General de Sociedades Mercantiles), en este texto se ha tomado como fecha oficial de su fundación el 31 de octubre de 1953. En este día los lasallistas registraron su editorial ante Hacienda y obtuvieron su Registro Federal de Contribuyentes (RFC), lo cual puede considerarse su fundación por la vía fiscal.

Aquí es necesario señalar que en México las obras pertenecientes a los religiosos de la Salle están divididas en dos distritos (actualmente llamados México-Norte y México-Sur Antillas) desde el año 1960. Pese a esto, la Editorial Enseñanza continuó proporcionando sus servicios a los Hermanos de ambas jurisdicciones por lo menos hasta mediados de la década de los setenta. De hecho, autores lasallistas del Distrito México-Norte siguieron publicando en Enseñanza (que quedó en manos de México-Sur) tiempo después del surgimiento de Editorial Didáctica en su distrito, un evento que también es complicado de fijar en el tiempo. Tradicionalmente, los Hermanos de la Salle sitúan el origen de esta editorial entre 1975-1980 pero, ante la dificultad de consultar el acta constitutiva que pudiera fijar la fecha de creación de esta empresa educativa, en este artículo se considera el 27 de agosto de 1991 (día en que la editorial fue registrada ante Hacienda y obtuvo su RFC) como la fecha oficial de su fundación, por lo menos por la vía fiscal. 
Ambas editoriales ya no se encuentran activas pero, indudablemente, estas empresas fueron fundamentales en los proyectos educativos de sus distritos, como un sostén económico y, sobre todo, como un medio para propagar sus ideas en materia educativa (G. Orozco García, S. A. Ávalos Cárdenas y A. Aranda Ramírez, comunicación personal, 12 de Octubre 2019).

Para contextualizar el fenómeno estudiado, hay que señalar también que el período seleccionado fue un momento de estabilidad para la historia lasallista en México, a diferencia de los primeros años que estuvieron marcados por las irregularidades de la Revolución, la persecución religiosa y la educación socialista (Grousset y Meissonier, 1983b).

Asimismo, hay que mencionar que la fecha final que delimita el lapso elegido para este artículo de investigación (1989), coincide con la publicación de la Carta a la Familia Lasallista (Consejo General, 1989), sin la cual no se puede comprender el devenir reciente de los Hermanos de las Escuelas Cristianas. Esto se debe a que esta carta está directamente enlazada con las discusiones y reflexiones que los religiosos de la Salle sostuvieron durante la segunda mitad del siglo XX (y que se pueden localizar en la documentación existente) sobre la necesidad de formar a sus laicos (alumnos, maestros y colaboradores) en varios aspectos como la educación cívico-política.

La elección de esta fecha de corte también se relaciona con la llegada de Carlos Salinas de Gortari a la presidencia de México, que provocaría algunos cambios vinculados a la ley de asociaciones religiosas y culto público en 1991, y reformaría los libros de la CONALITEG en 1992. Asimismo, dado que para este texto se está contemplando la labor de la Editorial Enseñanza, se consideró necesario finalizar el ciclo elegido antes del surgimiento oficial para este estudio de la Editorial Didáctica en 1991.

Sobre el Hermano Bernardo Zepeda Sahagún hay que decir que nació en Ejutla, Jalisco el 6 de junio de 1914, siendo el primer hijo de Herminio Zepeda Pelayo y María Sahagún de Zepeda. Del recorrido vital de este Hermano existen algunos datos sobre su ingreso al Colegio Francés La Salle de la Ciudad de México, una vez que concluyó su educación básica.

En enero de 1934, el Hermano Zepeda ingresaría al noviciado lasallista, si bien tuvo que proseguir su formación en Estados Unidos. Más adelante sería destinado al Colegio Cristóbal Colón (Ciudad de México) y al Colegio Benavente (Puebla), después de lo cual tomaría la dirección del Colegio Margil en Zacatecas (1952-1959). Posteriormente, Bernardo Zepeda Sahagún sería director del Colegio Miguel de Bolonia en San Juan de los Lagos, Jalisco (1959-1960, 1963-1967), director y subdirector del Colegio Regis en Hermosillo, Sonora (1960-1963 y 1967-1973 respectivamente), además de que trabajaría en el Colegio Febres Cordero de Guadalajara (1973 a 1978), ciudad en la que fallecería el 7 de septiembre de 1978, en el Hospital del Carmen.

A fin de proporcionar una perspectiva más amplia sobre este religioso de la Salle, es necesario apuntar que fue el autor de cerca de catorce libros de texto para primaria y secundaria de Ortografía, Historia General, Historia de América, Historia de México y Civismo (E. González Pérez, comunicación personal, 19 septiembre 2018). 


\section{El Modelo Tridimensional de Análisis Crítico del Discurso de Norman Fairclough}

Para la revisión de los libros de texto de civismo redactados por el Hermano Bernardo Zepeda Sahagún se propone una metodología que consta de dos pasos:

1. La descripción: Dado que el método de revisión propuesto es de carácter hermenéutico, el proceso descriptivo es una etapa fundamental.

2. El Modelo Tridimensional de Análisis Crítico del Discurso de Norman Fairclough: Implica analizar la evidencia aportada por los manuales escolares, visualizando al discurso como texto, como práctica discursiva y como práctica social (Rogers, 2011, p. x). Esto quiere decir que el discurso puede estudiarse "como unidad lingüística o 'pieza de lenguaje' escrito o hablado", considerando los “procesos de producción e interpretación de los textos”, además de analizar el "carácter situacional e institucional del evento discursivo" (Gómez Cuevas, 2015, p. 315).

\section{Del buen cristiano al ciudadano: Un estudio de la cortesía y la urbanidad en los libros de civismo de los Hermanos de la Salle en México}

\subsection{La descripción:}

El libro de civismo para estudiantes de $3^{\circ}$ y $4^{\circ}$ que Bernardo Zepeda Sahagún redactó está compuesto por 7 capítulos, mientras que el de $5^{\circ}$ y $6^{\circ}$ años posee doce. Cabe señalar que para los propósitos del presente artículo (enfocado en la formación de una conducta civilizatoria y de las buenas maneras) se emplearán los primeros 5 capítulos de ambos manuales escolares.

Los libros destinado a los alumnos de $3^{\circ}$ y $4^{\circ}$ comienzan con un saludo, al final del cual se invita al lector a ser “(...) amante hijo...buen estudiante...y más tarde...un perfecto ciudadano!”. En las páginas inmediatamente posteriores se da a entender al lector la necesidad de adentrarse en el civismo a través de una práctica comprometida pues "no es difícil aprender” los temas cívicos, "pero la práctica sí te será difícil", por lo cual se sugiere atender las enseñanzas "con buena voluntad y siguiendo al pie de la letra los consejos de tus padres y maestros, así como de las personas mayores” (Zepeda Sahagún, 1964, pp. 9, 13). Asimismo, en las primeras páginas del texto se establece claramente que se pretende normar la conducta del alumno y:

Crear en ti, hábitos de orden, de trabajo individual y colectivo, de disciplina, así como recto modo de proceder, urbanidad y buenas maneras, que te permitan actuar en la sociedad de que formas parte con un mayor sentido de cooperación y responsabilidad (Zepeda Sahagún, 1964, p. 14).

Más adelante se menciona también:

Tú puedes, si quieres, ser un hombre útil, haciendo que tu voluntad se decida en cualquiera circunstancia a seguir sin titubeos y con alegría el bien. 
(...) Recuerda y ten presente que siempre debes cumplir con tus obligaciones...las que encontrarás en los Mandamientos de la Ley de Dios y de la Iglesia, así como en las leyes civiles que rigen a nuestro país.

(...) Sí, una palabra: lee, estudia y practica lo que está en este librito. El civismo no es teoría sino práctica diaria de hoy, de mañana y de siempre (Zepeda Sahagún, 1964, p. 14).

En el caso del libro para $5^{\circ}$ y $6^{\circ}$ años, en las primeras páginas se menciona que "no es difícil aprender en teoría lo que enseña el civismo, pero lo que sí es difícil es la práctica de dichas enseñanzas (Zepeda Sahagún, 1965, p. 14). Asimismo, se señala que:

El carácter es el resultado de las arduas luchas de la educación de sí mismo, de abnegación sin límites, de una batalla espiritual sostenida virilmente.

(...) Para educar el carácter hay que luchar para adquirir ideales definidos, principios sólidos; en segundo lugar hay que acostumbrarse a obrar con un ejercicio continuo, a obrar según esos nobles ideales en cualquier circunstancia de la vida (Zepeda Sahagún, 1965, p. 14).

Una vez concluidos estos señalamientos, el libro pasa a la sección de la familia en la que se señalan con claridad los deberes que los hijos tienen para con sus padres (Zepeda Sahagún, 1964, pp. 23-24; Zepeda Sahagún, 1965 pp. 24-26), mientras que para los alumnos de $5^{\circ}$ y $6^{\circ}$ se hacen algunas recomendaciones extras sobre los deberes hacia los hermanos y el respeto a la mujer (Zepeda Sahagún, 1965, pp. 26-27).

Las costumbres constituyen el centro del siguiente apartado, el cual comienza con las siguientes palabras:

Por medio de la repetición de los mismos actos, ya sean buenos o malos, llegarás a adquirir hábitos o costumbres, buenos o malos.

De esto resulta que siempre y en todas partes debes obrar bien aunque nadie te vea, para que llegues a adquirir buenos hábitos.

Estos buenos hábitos harán de ti un buen hijo y un buen ciudadano, de quien no se avergonzarán ni tus padres ni tu patria. Entre los buenos hábitos que debes adquirir, y siempre, recuérdalo bien, por medio de la repetición de los actos a ellos concernientes, deben estar el aseo, el trabajo, el ahorro, el honor, la firmeza y la dignidad; la gratitud, la fidelidad, la lealtad, el compañerismo, el espíritu de justicia, etc (Zepeda Sahagún, 1964, p. 31; Zepeda Sahagún, 1965, p. 35).

Después de esta introducción, el texto se enfoca en hacer sugerencias relacionadas con el aseo que, de seguirse, propiciarán que el lector sea apreciado por otros y conserve su salud. Asimismo, la lectura promueve el trabajo y el ahorro, la gratitud, la fidelidad, la lealtad, la puntualidad, la responsabilidad, la discreción y la expresión manifiesta de la buena educación mediante un lenguaje adecuado y dejando de 
lado los chistes (Zepeda Sahagún, 1964, pp. 31-37; Zepeda Sahagún, 1965, pp. 35-41). Para todo esto, se pone de ejemplo a Jesucristo tal y como ocurre en los siguientes fragmentos:

Un favor se paga con otro favor. Si eres agradecido, el mostrar tu agradecimiento dispone a tu bienhechor a hacerte nuevos favores.

En cierta ocasión, nos cuenta el Santo Evangelio, se presentaron diez leprosos, ante N. S. J. C., quien los curó después de enviarlos ante el sacerdote, que era el único capacitado para certificar su curación. De los diez, únicamente un samaritano volvió a dar gracias a Jesús del favor que le había hecho. Este era agradecido, los otros nueve fueron unos ingratos o malagradecidos

Y también:

Trabaja, ejecuta siempre los actos buenos con la mira de adquirir la costumbre, el hábito de hacer el bien, así, solamente así, tendrán su recompensa, si no en este mundo, sí en el otro, en donde N. S. J. C. te recibirá con los brazos abiertos y te dirá: "Ven siervo bueno y fiel, entra en el gozo de tu Señor" (Zepeda Sahagún, 1964, pp. $35,37)$.

El capítulo que se considera específicamente enfocado en la conducta otorga al lector una serie de consejos centrados en el buen comportamiento en casa o durante la conversación (saludar antes de hablar, no alzar la voz y mantener un ritmo adecuado al expresarse); a la hora de sentarse a la mesa (no hacerlo antes que los padres o los mayores, no apoyar el antebrazo ni los codos en la mesa, no sorber, etcétera), y la conducta en la calle (moverse en ella con decoro siendo respetuoso con otros transeúntes y sacrificando la propia comodidad de ser necesario, caminar por el lado derecho de la acera, no correr, etcétera) y en los centros de espectáculos (como no molestar a otros asistentes y evitar hacer ruidos) (Zepeda Sahagún, 1964, pp. 43-50; Zepeda Sahagún, 1965, pp. 47-55).

Para los alumnos de $5^{\circ}$ y $6^{\circ}$ hay algunos temas extra como la conducta en oficinas públicas, los consultorios, los despachos, los almacenes, las tiendas, los bazares y los viajes. Así, a estos estudiantes se les invita a no dar la espalda al escenario y al público en un palco, o en el caso del comportamiento en las oficinas públicas se recomienda, por ejemplo, respetar los turnos de atención y exponer brevemente el motivo de la diligencia. Las mismas sugerencias se hacen para la visita a consultorios y despachos, con el agregado de que se debe pagar sin regatear (Zepeda Sahagún, 1965, pp. 51-53)

Para los libros de $5^{\circ}$ y $6^{\circ}$, en los almacenes se debe mantener una actitud educada a la hora de solicitar lo que se va a comprar y disculparse en caso de no adquirir algo. En el caso de los viajes, en la lectura se sugiere llegar con algunos minutos de anticipación cuando se viaja con otros, hacer conversación, pero sólo cuando la persona de mayor rango inicia la charla o no fumar sin pedir permiso (Zepeda Sahagún, 1965, pp. 51-53).

En el apartado correspondiente a la escuela se invita a ser sincero con los compañeros, no tocar ni tomar sus pertenencias sin permiso, no envidiar o tener mala voluntad a los estudiantes más adelantados y más bien esforzarse por igualarles en talento y estudios y, en general, tratar de ganarse su aceptación 
y simpatía a través de la bondad. También se sugiere cooperar con la dinámica general de la clase y presentar un buen comportamiento en ausencia del docente (Zepeda Sahagún, 1964, pp. 54-55; Zepeda Sahagún, 1965, pp. 61-63).

En el trato con el maestro, Bernardo Zepeda Sahagún sugiere, por caridad, gratitud y respeto, no hablar mal del profesor y no disgustarse al ser corregido. Para el texto es importante también que los niños y jóvenes saluden y cedan el paso a profesores y superiores, no dañen el inmueble ni el mobiliario, no corran (más bien que anden "con naturalidad, pero sin lentitud ni precipitación”); y en el recreo no griten y eviten los juegos de manos (Zepeda Sahagún, 1964, pp. 56-59; Zepeda Sahagún, 1965, pp. 63-65). Con relación a los deportes y otros eventos sociales de la escuela se menciona:

Recuerda que los juegos y deportes ayudan a conservarse en buena salud y ofrecen múltiples oportunidades para dominarse, para practicar las reglas de caballerosidad y de urbanidad sobre las que descansan las buenas relaciones. Debes tener para tus compañeros de juegos mucha condescendencia, solidaridad, justicia y respeto. En los juegos hay que jugar, sí, y también querer ganar, pero, si a pesar de todo el juego se pierde, hay que saber perder.

Sigue en todos los deportes las indicaciones de los encargados de ello, cumple siempre con tus compromisos y juega siempre limpio...

Caballerosidad ante todo...en los juegos también debes mostrar que eres educado, aun cuando tus contrincantes no lo sean (Zepeda Sahagún, 1964, p. 59).

\subsection{Los libros de texto de Bernardo Zepeda Sahagún como práctica discursiva}

Para esta fase de análisis es necesario adentrarse en la producción e interpretación de los textos. Este paso implica entender a los manuales escolares lasallistas como una herramienta pedagógica y cognoscitiva de gran relevancia que participa en el proceso de enseñanza-aprendizaje, es decir, que son textos de carácter educativo, por lo que median los procesos formativos de estudiantes y docentes (Partido Calva, 2007, p. 2). Para perfilar esto mejor hay que añadir que se pueden considerar textos de carácter expositivo, que poseen funciones informativas, es decir, que buscan "alcanzar la apropiación del mundo real"; apelativas, pues entre sus intenciones se encuentra la de modificar el comportamiento del lector a través de la cortesía y la persuasión, más que de órdenes estrictas y directas (Carreón Cruz, 2004).

Puede considerarse que los libros de civismo que los Hermanos de la Salle publicaron en México marcaron un camino y un enfoque en los procesos de construcción del conocimiento porque, además, como ya se ha señalado, poseían una fundamentación teórica (Partido Calva, 2007, p. 3), pedagógica y religiosa que, muy probablemente, se hizo sentir en las aulas de los centros educativos de este instituto religioso. Posiblemente esto se vio favorecido por una situación en la que el libro de texto no competía o se complementaba con otros recursos pedagógicos (como sucede actualmente), por no hablar del protagonismo que estos manuales escolares debieron tener dentro del salón de clase y fuera de él, un papel que el libro de clase posee de por sí, innegablemente, dada su naturaleza como "uno de los soportes más fieles 
del currículo escolar ejercido" y "guía privilegiada para su ejecución”, sin olvidar que son trasmisores de contenidos culturales e ideológicos y agentes fuera de la esfera estrictamente escolar (Molina Puche M. et al., 2010, p. 1).

Para reconstruir de una mejor manera los procesos de interpretación de los textos contenidos en los libros de Bernardo Zepeda Sahagún hay que centrarse necesariamente en los destinatarios de estos manuales escolares que, por su edad, son personas inexpertas, que se acercaban al libro de clase como "fuente incuestionable del saber", es decir que existe asimetría en las relaciones con el lector (Martín Rojo, 2003 , p. 164). Con esto puede vislumbrarse la importancia que tuvo el libro de texto en la manera en que los estudiantes lasallistas pudieron percibir la realidad que les rodeaba (Atienza Cerezo, 2007, p. 546).

\subsection{Los libros de texto de civismo lasallistas como práctica social}

Michel de Certeau señaló que para las órdenes religiosas su tradición es un elemento que ejerce gran influencia en el presente en el que realizan sus acciones (2006, pp. 71-74). Esta idea resulta especialmente útil para comprender el carácter situacional e institucional de los libros de texto lasallistas que son de interés para este artículo, pues estas ideas permiten plantear dos vías de análisis: la influencia de la Iglesia (y la religión) Católica y el respeto (lo que implica una constante adecuación) por la espiritualidad de Jean-Baptiste de la Salle.

La primera de estas sendas revela un propósito para los libros de civismo de los Hermanos de la Salle en particular y para su instrucción cívica en general: promover y formar un tipo de alumno, una idea de persona que encuentra su fuente de inspiración en los valores del Evangelio y su modelo en Jesucristo (con lo cual cobran sentido las breves alusiones a este personaje que aparecen en los manuales escolares de Zepeda Sahagún).

Esto significa, a su vez, que la formación cívica de los religiosos de la Salle debe vincularse con las enseñanzas de la Iglesia, lo cual se tradujo en el uso de las ideas que aparecerían en el Catecismo de la Iglesia católica (es decir, el cuerpo estructurado que posee la finalidad de explicar el contenido dogmático de la fe católica, el cual fue presentado durante el pontificado de Juan Pablo II) como una fuente para sus manuales escolares de civismo.

De manera puntual (y gracias a la revisión descriptiva que se hizo líneas arriba) se puede afirmar el notable peso que lo establecido por la Iglesia acerca de la virtud tuvo en los libros de civismo aquí estudiados:

La virtud es una disposición habitual y firme a hacer el bien. Permite a la persona no sólo realizar actos buenos, sino dar lo mejor de sí misma. Con todas sus fuerzas sensibles y espirituales, la persona virtuosa tiende hacia el bien, lo busca y lo elige a través de acciones concretas.

Y también:

Las virtudes humanas adquiridas mediante la educación, mediante actos deliberados, y una perseverancia, mantenida siempre en el esfuerzo, son purificadas y eleva- 
das por la gracia divina. Con la ayuda de Dios forjan el carácter y dan soltura en la práctica del bien. El hombre virtuoso es feliz al practicarlas (Catecismo de la Iglesia católica, 2018, parra. 1803, 1810).

Estas referencias pueden verse a lo largo de los libros de Bernardo Zepeda Sahagún, cuando se insiste en la importancia de practicar el bien y lo difícil de hacer del civismo y las buenas maneras una constante en la vida, ante lo cual es necesario ejercitar estas enseñanzas de manera permanente. Con base en esto puede decirse que el civismo en los centros de los Hermanos buscaba formar un niño virtuoso, que tuviera la capacidad de ser prudente en sus acciones y sus palabras; justo; con fortaleza, expresada en su constancia y su firmeza ante las situaciones cotidianas; con un carácter orientado por la templanza para renunciar a su propia comodidad en beneficio de los demás (y para tomar las mejores decisiones en aspectos de la vida como el trabajo, el ahorro, las diversiones, etcétera, además de formar la voluntad); y con fe, esperanza y caridad para guiar sus acciones.

Los textos bíblicos (Ex 20: 12) y el catecismo se hacen presentes en los Diez Mandamientos, que también aparecen entre las enseñanzas de los libros de clase de Bernardo Zepeda Sahagún. De manera concreta es el cuarto mandamiento el que resulta más evidente cuando se hacen recomendaciones hacia el niño para el trato con sus padres. Sin embargo, la amabilidad, la cortesía y el respeto se extienden también hacia los miembros de la familia, los maestros, jefes y funcionarios pues, de acuerdo con el Catecismo de la Iglesia (que si bien apareció hasta finales del siglo XX -concretamente en 1992 en su versión en francés y la versión latina oficial de 1997- se usa como referencia en este artículo):

El cuarto mandamiento se dirige expresamente a los hijos en sus relaciones con sus padres, porque esta relación es la más universal. Se refiere también a las relaciones de parentesco con los miembros del grupo familiar. Exige que se dé honor, afecto y reconocimiento a los abuelos y antepasados. Finalmente se extiende a los deberes de los alumnos respecto a los maestros, de los empleados respecto a los patronos, de los subordinados respecto a sus jefes, de los ciudadanos respecto a su patria, a los que la administran o la gobiernan.

Este mandamiento implica y sobreentiende los deberes de los padres, tutores, maestros, jefes, magistrados, gobernantes, de todos los que ejercen una autoridad sobre otros o sobre una comunidad de personas (Catecismo de la Iglesia católica, 2018, parra. 2199).

Esto se encuentra en los libros de civismo de los Hermanos de la Salle en México cuando se afirma que "todos deben tener presente que en el cuarto mandamiento no solamente se manda honrar a los padres sino también a los legítimos superiores, porque todo superior ha sido constituido por Dios sobre los inferiores" (Zepeda Sahagún, 1965, p. 26). Con esto puede afirmarse que la educación cívica lasallista buscaba formar niños obedientes, respetuosos y responsables con la Iglesia, la Familia, el Estado, la sociedad y otras instituciones, aunque también es evidente que estas ideas ayudaban a perpetuar el estado de las cosas. 
Aquí es fundamental reconocer las limitaciones en el uso de libros de texto como fuentes, pues siempre habrá una dificultad para reconstruir la realidad del trabajo en el aula. Pese a ello, existen un número limitado de referencias en la documentación existente sobre los religiosos de la Salle, que dan cuenta de las reflexiones que los Hermanos hacían sobre este tipo de cuestiones con el fin de ponerlas en práctica en las dinámicas de sus centros escolares.

Algunas de estas ideas reconocen que el niño posee una naturaleza voluble, que lo hace sujeto a ser instruido en el orden y la disciplina, manifiestos en los buenos hábitos físicos (como la higiene y el "desarrollo racional del vigor") y el perfeccionamiento moral e intelectual. El Hermano y el docente tenían pues, un papel vital en el desarrollo de este tipo de conductas mediante estrategias como la preparación de los cursos con esmero, la clasificación de los estudiantes de acuerdo a su comportamiento, una distribución de las materias y del tiempo de trabajo en clases acorde a lo que se esperaba lograr en el alumno y la creación de un ambiente que fortaleciera la disciplina interior mediante actividades que fomentaran una cultura del esfuerzo. Otros medios directos que los lasallistas propusieron para el desarrollo de la autoregulación y la modificación de las conductas fueron la necesaria serenidad y el prestigio del maestro (que se consideraban fundamentales para el orden de los alumnos en el salón de clases) y la regularidad en la realización de las tareas, la exactitud de los horarios, el aseo personal y del entorno, etcétera (Parra A., 1967, pp. 6-8).

Otro testimonio, que fue motivado por las Olimpiadas de 1968, ofrece una ventana para observar la manera en la que los Hermanos aterrizaron sus ideas sobre la cortesía y las buenas maneras de forma cotidiana. Así, después de que el Distrito México-Norte decidiera elaborar unos textos sobre trato social (Hermanos de las Escuelas Cristianas, 1967, p. 3) en un artículo en concreto se llegó a recomendar la elaboración de campañas "Pro Urbanidad" y "Pro Buenas Maneras", o bien, la realización de una clase de urbanidad al menos una vez a la semana, con apoyo de la catequesis y las reflexiones, a fin de transmitir a los alumnos la importancia de la virtud y de la dignidad humana (Ramírez S., 1967, pp. 25-26).

Para los lasallistas la motivación para una campaña de este tipo radicaba en el hecho de que las nuevas generaciones habían dejado de lado la urbanidad y la cortesía por considerarlas falsas o poco relevantes (Ramstone, 1967, p. 18). Pero, para los Hermanos, si sus propósitos de trasladar las buenas maneras al salón de clase se llevaban a cabo, traerían como consecuencia un buen comportamiento en los alumnos pues, de acuerdo con los religiosos de la Salle "tratar bien a nuestro prójimo es un deber, no tan sólo de buen Mexicano, sino de "cristiano"', un signo de cortesía cristiana, muy diferente a las políticas seguidas por el Estado mexicano que, a juicio de los lasallistas, se enfocaba únicamente en la entrada de divisas al país. Así pues, aprovechando las olimpiadas y las visitas de extranjeros, se pedía:

Dar informes corteses a extraños y compatriotas.

Corregir cortésmente al que se equivoque.

Tratar con amabilidad y cortesía.

Contribuir y dar hospitalidad al necesitado

Hacer que el visitante se sienta en México como en su propio hogar. 
Aceptar al turista como es y no como quisiéramos que fuera.

Hacer que el turista se lleve la mejor impresión de México.

Tratar al extranjero con honradez y evitar el explotarlo y o querer sacar provecho económico exagerado de él.

No burlarse del turista por su desconocimiento de algunas cosas.

Finalmente, a lavar y a barrer nuestras banquetas y a recoger la basura, para que borremos el apelativo que nos habían endilgado "nuestros primos" del Norte, "Greasers"=Mugrientos (Ramírez S., 1967, pp. 25-27).

Este fragmento en particular tiene una clara influencia, nuevamente, de los textos bíblicos (Tb 4, 5-11; Is 58, 6-7; Mt 6, 2-4; Mt 25, 31-46; Lc 3, 11; Lc 11, 41; Hb 13, 3; Sant 2, 15-16; Sant 2, 17-22) y de lo que posteriormente fue el Catecismo, pues es una adecuación de las llamadas Obras de Misericordia corporales y espirituales (Catecismo de la Iglesia católica, 2018, para. 2447).

La segunda vía para entender el carácter situacional e institucional de los libros de texto lasallistas, la espiritualidad de Jean-Baptiste de la Salle, se manifiesta a través de las buenas maneras, es decir, las expresiones de comportamiento concretas que se derivan de la misión evangélica arriba explicada y del proyecto civilizador del Fundador de los Hermanos de las Escuelas Cristianas. Esto puede verse en las indicaciones a la hora de comer, en las sugerencias para saludar, en el comportamiento en clase, etcétera.

Aunque la presencia de las buenas maneras también obedece a la necesaria gradualidad del proceso educativo. Esto significa que, debido a las edades y los grados de madurez de los niños, se empleó la cortesía y la civilidad como medios para fomentar el sentido comunitario, el respeto, la bondad y otras conductas igualmente relevantes desde la óptica católica. Por otra parte, no hay que descartar la posibilidad de que las buenas maneras fueran también un medio para una asimilación gradual de los valores que los lasallistas consideraban importantes.

Siguiendo esta línea de pensamiento, la formación cívica que promovían los Hermanos de la Salle era una herramienta que se proponía inducir al estudiante a hacer el bien, aprovechando cada situación social para vincularse cordialmente con otros miembros de su entorno y ejercer la caridad, en todo lo cual puede verse la influencia del concepto de la persona humana (Catecismo de la Iglesia católica, para. 1701-1707).

Tomando en cuenta todos los elementos arriba expuestos se puede concluir que para el proyecto educativo lasallista, el propósito fundamental radicaba en formar buenos cristianos que, en un segundo paso, serían buenos ciudadanos, y no al revés. Esto significa que para este tipo de educación el ejercicio pleno, decidido y comprometido de la fe es la causa primera del deber de participar en el universo de lo político y de la responsabilidad frente a la vida pública (si bien estos manuales escolares no preveían una ruptura del orden, las instituciones y las autoridades establecidos). Ciertamente, estas ideas ofrecen matices frente a la ciudadanía promovida por el sistema educativo del Estado, por lo que se propone la categoría de persona cívica (y no ciudadano) para designar el modelo de ciudadanía que los centros educativos lasallistas estaban abocados a formar. 


\section{Conclusiones}

A través de los libros de civismo para primaria que los Hermanos de las Escuelas Cristianas publicaron en México entre 1953-1989 (fundamentados en la doctrina católica y la espiritualidad lasallista) se buscaba modelar un niño virtuoso, obediente, respetuoso y responsable con las instituciones, es decir, una persona cívica. Ante esto, es posible visualizar la propuesta cívica lasallista como un proyecto enfocado en el desarrollo y el fortalecimiento de la dimensión apostólica de los seglares que acudían a sus colegios, a la vez que no se proponía una ruptura con el orden establecido del mundo y la sociedad.

Esta conclusión abre nuevas interrogantes sobre las maneras en las cuales la formación cívica recibida en los colegios de los Hermanos de la Salle pudo vincularse con tres fenómenos del período comprendido entre 1953-1989 que se relacionan entre sí y que han podido localizarse en la documentación existente: La implementación de la Acción Católica en los Colegios Lasallistas (concretamente la Juventud Estudiante Católica-JEC-), el surgimiento de lo que hoy se conoce como el lasallismo (un fenómeno que se desarrolló casi de manera simultánea en México y en el mundo, tal y como lo sugiere la evidencia encontrada) y el desarrollo de los grupos apostólicos -catecismos, círculos vocacionales, etc.- (Hermanos de las Escuelas Cristianas, 1959a, p. 10; Hermanos de las Escuelas Cristianas, 1959b, p. 14, Hermanos de las Escuelas Cristianas, 1960, pp. 15-20, Hermanos de las Escuelas Cristianas, 1961, pp. 12-14).

Otra posible vía de investigación para el futuro debe enfocarse necesariamente en los exalumnos y las asociaciones lasallistas. En este sentido, sería interesante conocer las maneras en las cuales la formación cívica recibida en los colegios de los Hermanos de la Salle se transformó (o no, pues hay que contemplar también esa posibilidad) en un medio para la agencia política y social como ya se ha estudiado, por ejemplo, sobre la participación lasallista en Puebla en los enfrentamientos entre diversos grupos de la Benemérita Universidad Autónoma de Puebla (Dávila Peralta, 2003; Yáñez Delgado, 2000).

Los resultados de esta indagación podrían abonar al estudio del pensamiento y la participación de los católicos mexicanos, además de que podrían compararse con la labor hecha por otras órdenes religiosas e, incluso, con el trabajo realizado por parte de otros grupos religiosos no católicos. Muy necesario también es el rastreo de testimonios que permitan reconstruir las prácticas educativas concretas que los religiosos lasallistas llevaron a cabo en México. Esta labor puede enfocarse en todas las etapas de formación y en las diversas áreas de conocimiento, entre ellas el civismo.

En este punto de las conclusiones es necesario señalar que los vacíos historiográficos no son suficientes para justificar la realización de una investigación como la del presente artículo, pues también hay que atender a las necesidades del entorno, y buscar soluciones a sus problemáticas a través de la investigación. En este sentido, la mirada hacia al pasado de la enseñanza cívica de los Hermanos de las Escuelas Cristianas permite proponer temas y estrategias a implementar en la formación cívica de las escuelas, partiendo de una perspectiva específica: la reflexión y la acción ciudadana relacionada con el medio ambiente.

Como se ha visto en líneas anteriores, la dimensión humana y social de la educación cívica se hace sentir en los libros de texto de los Hermanos de la Salle y en otros proyectos educativos. Sin embargo, ésta debe necesariamente complementarse con un enfoque que tome en consideración al mundo natural. 
Y es que la ciudadanía es un concepto muy complejo que consiste en derechos y obligaciones que, a su vez, son fluidos, lo que permite que esta noción evolucione hasta convertirse en un vehículo crucial para la toma de conciencia y el cambio de comportamiento hacia el medio ambiente (Latta, 2014, pp. 38-39). Para ello, claro está, la formación cívica deberá concebirse como un conocimiento sensible a y con injerencia en todas las esferas de la vida de las personas, incluyendo el entorno natural.

De esta manera, así como los cursos de civismo acentúan la formación del alumno en los derechos humanos, esta educación deberá promover el derecho a un ambiente sano (el cual, por cierto, es un derecho de tercera generación, plenamente reconocido por diversas instituciones y estados) (Hernández, 2012, p. 494)- como otro aspecto fundamental de la enseñanza en el aula. Asimismo, la participación y la agencia políticas deberán ser enfocadas también hacia las acciones para con el medio ambiente lo cual, por cierto, incide en indicadores sociales y ayuda a cambiar las condiciones injustas en las cuales viven muchas personas (Certomà y Martellozzo, 2019).

Para ello será necesario también un conocimiento de las normas y las instituciones relacionadas con este tipo de temas, que deberá estar acompañado de la praxis a través de las actividades con los estudiantes. Éstas últimas deberán basarse en problemas ciudadanos y medioambientales reales que, cuando la edad de los alumnos lo permita, pueden traducirse en acciones concretas para cuestiones que afecten su entorno más cercano.

\section{Agradecimientos}

A todos los profesores del programa de doctorado en Historia de la Universidad de Guanajuato, en especial a los Doctores Graciela Velázquez Delgado, Miguel Ángel Segundo Guzmán, Miguel Ángel Guzmán López y Gerardo Martínez Delgado. Al Doctor Miguel Ángel Hernández Fuentes, director de tesis; y los Doctores Valentina Torres Septién, María Guevara Sanginés, Ramiro Jaimes Martínez y José Elías Guzmán López, miembros del Comité Tutorial. Un especial reconocimiento para María Fabiola Marín Andrade (Asistente de la Coordinación de la Maestría y Posgrados en Historia) y todos los bibliotecarios de la Biblioteca Luis Rius de la Universidad de Guanajuato.

Un agradecimiento también para todos los miembros del Instituto de los Hermanos de las Escuelas Cristianas y de las Hermanas Guadalupanas De La Salle (y a los seglares que trabajan con ellos en su proyecto educativo como el Ing. Rafael Ángel Bonilla Lira) por su colaboración para la realización de este trabajo. Especialmente a los Hermanos Lorenzo González Kipper, Jesús Alberto Rubio Názer, Paul Aubin, Juan Ignacio Alba Ornelas, Adalberto Aranda Ramírez, Fernando Alvarado Hernández, Enrique González Pérez, Guilebaldo Orozco García, Sergio Armando Ávalos Cárdenas, Jorge Gallardo de Alba, las comunidades de la Casa Generacilia, de la Casas Centrales de los Distritos México-Norte, México Sur-Antillas y del Noviciado Interdistrital Nuestra Señora de Lourdes.

Muchas gracias a Valeria Alejandra Olivares Olivares y Juan Camilo Riobó Rodríguez, Doctores en Historia por la Universidad de Guanajuato y, finalmente, a Marusia González Martínez, Blanca Estela Martínez Rodríguez y Eduardo Varela Valdés. 


\section{Financiamiento}

Esta investigación se llevó a cabo gracias a una Beca Nacional CONACYT para estudiar en el Doctorado en Historia de la Universidad de Guanajuato, perteneciente al Programa Nacional de Posgrados de Calidad (PNPC) con el proyecto: Educar buenos cristianos, para forjar buenos ciudadanos: Los Hermanos de la Salle en México y la formación cívica, 1953-1989.

\section{Referencias}

Arruñada, B. (2010). Protestants and Catholics: Similar Work Ethic, Different Social Ethic. The economic Journal, 120 (547), 890-918. http://doi.org/10.1111/j.1468-0297.2009.02325.x

Atienza Cerezo, E. (2007). Discurso e ideología en los libros de texto de ciencias sociales. Discurso y Sociedad, 1 (4), 543-574. https://repositori.upf.edu/bitstream/handle/10230/23523/Atienza_dis_disc. pdf? sequence $=1 \&$ isAllowed $=\mathrm{y}$

Bryson, A. (2002). De la cortesía a la civilidad. Códigos cambiantes de conducta en la Inglaterra Moderna. En Torres Septién, Valentina (2002). Producciones de sentido: el uso de las fuentes en la historia cultural (355-363). Universidad Iberoamericana.

Calsamiglia Blancafort, H. y Tusón Valls, A. (1999). Las cosas del decir. Manual de análisis del discurso. Editorial Ariel.

Carreón Cruz, E. G. (2004). Clasificación de los diferentes tipos de textos y su uso en la educación primaria [PDF]. Universidad Pedagógica Nacional. http://200.23.113.51/pdf/23546.pdf

Certomà, C. y Martellozzo, F. (2019). Cultivating urban justice? A spatial exploration of urban gardening crossing spatial and environmental injustice conditions. Applied Geography, 106, 60-70. http:// doi.org/10.1016/j.apgeog.2019.03.007

Cervantes-Ortiz, L. (2007). La ética calvinista: una introducción a sus aspectos teóricos y prácticos. Teología y cultura, 8, 35-45. https://teologiaycultura.ucel.edu.ar/la-etica-calvinista-una-introduccion-a-sus-aspectos-teoricos-y-practicos/

Consejo General. (1989). Carta a la Familia Lasallista. Hermanos de las Escuelas Cristianas.

Dávila Peralta, N. (2003). Las santas batallas, la derecha anticomunista en Puebla, 2a edición. Gobierno del Estado de Puebla/ Benemérita Universidad Autónoma de Puebla.

De Certeau, M. (2006). El Mito de los Orígenes. En De Certeau, Michel (2006). La debilidad de creer. Katz.

De la Salle, J. B. (2001). Guía de las Escuelas Cristianas. En Valladolid, José María (editor) (2001). Obras completas de San Juan Bautista de la Salle. San Pío X, 2001.

De la Salle, J. B. (2001b). Reglas comunes de los Hermanos de las Escuelas Cristianas. En Valladolid, José María (editor) (2001). Obras completas de San Juan Bautista de la Salle. San Pío X, 2001. 
De la Salle, J. B. (2001c). Reglas de cortesía y urbanidad cristiana para uso de las Escuelas Cristianas. En Valladolid, José María (editor) (2001). Obras completas de San Juan Bautista de la Salle. San Pío X, 2001.

Elias, N. (2012). La sociedad cortesana. Fondo de Cultura Económica.

Elias, N. (2016). El proceso de la civilización: Investigaciones sociogenéticas y psicogenéticas. México: Fondo de Cultura Económica.

Fairclough, N. y Wodak, R. (2000). Análisis Crítico del Discurso. En Van Dijk, Teun A. (Compilador) (2000). El discurso como interacción social. Estudio del discurso: introducción multidisciplinaria, (v. 2. , pp. 367-404), Gedisa.

Gómez Cuevas, H. (2015). Análisis crítico del discurso al campo del currículum de la formación inicial docente en Chile. Estudios pedagógicos, XLI (1), 311-322. https://www.redalyc.org/articulo. $\underline{\text { oa? id }=173541114018}$

Grousset, B. A. y Meissonier, A. (1983a). La Salle en México. Primera Etapa 1905-1921. Sembradíos arrasados en espera de resurrección. Editora de Publicaciones de Enseñanza Objetiva.

Grousset, B. A. y Meissonier, A. (1983b). La Salle en México. Tercera Etapa 1947-1980. La expansión. México: Editora de Publicaciones de Enseñanza Objetiva.

Hermanos de las Escuelas Cristianas. (1959a). Avisos y recomendaciones. Quinta convención de antiguos alumnos lasallistas. La Salle en México, (9), 10.

Hermanos de las Escuelas Cristianas. (1959b) ¿Qué es el lasallismo? La Salle en México, (11), 14.

Hermanos de las Escuelas Cristianas. (1960). Gran convención lasallista en Roma. La Salle en México, (22), 15-20.

Hermanos de las Escuelas Cristianas. (1961). Movimientos apostólicos. La Salle en México, 12-14.

Hermanos de las Escuelas Cristianas. (1967). Plan para 'La Salle en México-Norte' 1967-1968. La Salle en México Norte, (104), 3.

Hernández, G. (2012). La educación de personas jóvenes y adultas y el derecho a la educación. El tema del sujeto juvenil. Revista Mexicana de Investigación Educativa, 17 (53), 485-511. https://www. redalyc.org/articulo.oa?id $=14023105008$

Iglesia católica. (2018). Catecismo de la Iglesia católica. Obra Nacional de la Buena Prensa.

Latta, A. (2014). Ciudadanía ambiental y las políticas de espacio y escala en Latinoamérica. Avá. Revista de Antropología, (24), 37-55. https://www.redalyc.org/articulo.oa?id=169039924002

López Sánchez, O. (2009). Virtuoso, templado y ahorrativo. Las prescripciones metodistas e higienistas del cuerpo en México, siglos XIX y XX. Op. Cit., (19), 179-210. https://revistas.upr.edu/index.php/ opcit/article/view/8022

Martín Rojo, L. (2003). El análisis crítico del discurso. Fronteras y exclusión social en los discursos racistas. En Íñiguez, Lupicinio (2003). Análisis del discurso. Manual para las ciencias sociales (157-201). Editorial UOC. 
Molina Puche M., S. et al. (2010). El uso del libro de texto en el aula de historia de segundo de bachillerato. II Congrés Internacional de DIDÀCTIQUES. Universitat de Girona, Departament de Didàctiques Específiques. https://dugi-doc.udg.edu/bitstream/handle/10256/2868/347.pdf?sequence=1

Parra A., V. (1967). “El orden y la disciplina”. La Salle en México Norte, (105), 6-8.

Partido Calva, M. (2007). Los libros de texto en la escuela primaria y sus implicaciones en la lectura. CPU-e, Revista de Investigación Educativa, (5), 1-21. Recuperado de https:/www.redalyc.org/ pdf/2831/283121712003.pdf

Ramírez S., J. M. (1967). Olimpíadas. La Salle en México Norte, (105), 25-27.

Ramstone. (1967). Olimpiadas. La Salle en México Norte, (107), 18-19.

Rogers, R. (editor). (2011). An introduction to Critical Discourse Analysis in Education. Routledge.

Torres Septién, V. (2002). Los códigos de cortesía como fuentes para la escritura de la historia. En Torres Septién, Valentina (2002). Producciones de sentido: el uso de las fuentes en la historia cultural (355-363). Universidad Iberoamericana.

Van Dijk, T. (2003). La multidisciplinariedad del análisis crítico del discurso: un alegato en favor de la diversidad. En Wodak, Ruth y Meyer, Michael (Compiladores) (2003). Métodos de análisis crítico del discurso (143-177). Gedisa.

Yáñez Delgado, A. (2000). La manipulación de la fe. Fúas contra carolinos en la universidad poblana, $2^{a}$ edición. Puebla: Imagen Pública y Corporativa.

Zepeda Sahagún, B. (1964). Y yo te digo, Civismo para $3^{\circ}$ y $4^{\circ}$ años de Primaria. Editorial Enseñanza.

Zepeda Sahagún, B. (1965). Un paso hacia arriba, Civismo para $5^{\circ}$ y $6^{\circ}$ años de primaria. Editorial Enseñanza. 